\title{
1 A diffusion-like process enables expansion of advantaged 2 gene mutations in human colonic epithelium
}

4 Cora Olpe ${ }^{1,2}$, Doran Khamis ${ }^{7}$, Maria Chukanova ${ }^{1}$, Richard Kemp ${ }^{1}$, Kate Marks ${ }^{4}$,

5 Cerys Tatton ${ }^{1}$, Cecilia Lindskog ${ }^{6}$, Nefeli Skoufou-Papoutsaki ${ }^{1,2}$, Anna Nicholson ${ }^{1}$,

6 Roxanne Brunton-Sim ${ }^{3}$, Shalini Malhotra ${ }^{5}$, Rogier ten Hoopen ${ }^{5}$, Rachel Stanley ${ }^{3}$,

7 Doug Winton ${ }^{1,2}$, Edward Morrissey ${ }^{7}$

8

9
${ }^{1}$ Cancer Research-UK Cambridge Institute, Li Ka Shing Centre, Robinson Way, Cambridge, CB2 ORE, UK

${ }^{2}$ Wellcome Trust-Medical Research Council, Cambridge Stem Cell Institute, Cambridge, UK

${ }^{3}$ Norwich Research Park BioRepository. James Watson Road, Norwich, NR4 7UQ, UK

${ }^{4}$ Pathology \& Data Analytics, Level 4, Wellcome Trust Brenner Building, St James's University Hospital, Beckett Street, Leeds, LS9 7TF, UK

${ }^{5}$ Department of Oncology, Box 231, University of Cambridge, Cambridge, CB2 2QQ, UK

${ }^{6}$ Department of Immunology, Genetics and Pathology, Science for Life Laboratory, Rudbeck Laboratory, Uppsala University, Uppsala, SE 751 85, Sweden

${ }^{7} \mathrm{MRC}$ Weatherall Institute of Molecular Medicine, University of Oxford, John Radcliffe Hospital, Headington, Oxford OX3 9DS, UK.

${ }^{*}$ Correspondence to:

Lead contact:

Dr. Douglas J. Winton

Email: doug.winton@cruk.cam.ac.uk

Dr. Edward Morrissey

Email:edward.morrissey@imm.ox.ac.uk

Ph: +44 (0) 1223769783 


\section{Significance statement}

43 Colorectal cancer is thought to arise from outgrowth of colonic crypts when genetic

44 alterations exceed a threshold. Therefore, quantification of the mode and dynamics

45 of clonal expansion within this tissue is key to understanding disease initiation. Here,

46 loss of KDM6A is found to increase the rate of crypt fission to generate large clonal

47 expansions in human colon with age. Crypt fusion is investigated as a means of

48 accommodating newly generated crypts. Homeostatic rates of fusion are inferred but

49 are not elevated within $\mathrm{KDM}^{-}$expansions. Rather we describe that the necessary

50 space can be generated by a crypt diffusion-like process. A diffusion coefficient is

51 estimated and used to define a threshold fission rate beyond which the balance

52 between the two processes is disrupted, that may represent a physical mechanism

53 for polyp growth.

\section{Abstract}

56 Colorectal cancer is thought to arise when the mutational burden of the clonal 57 population of stem cells within a colonic crypt exceeds a certain threshold. 58 Therefore, quantification of the fixation and subsequent expansion of somatic 59 mutations in histologically normal epithelium is key to understanding colorectal 60 cancer initiation. Here, using immunohistochemistry, loss of the histone demethylase $61 \mathrm{KDM6A}$ in normal human colonic epithelium is visualised. Interpretation of the age62 related behaviour of KDM6A-negative clones revealed significant competitive 63 advantage in intra-crypt dynamics. Further, subsequent clonal expansion into multi64 crypt patches was quantified to reveal a significant 5 -fold increase in crypt fission 65 rate. To accomodate the local accumulation of new crypts, the role of crypt fusion 66 was considered. However, no compensatory increase in fusion rate was found. 67 Instead, evidence for crypt diffusion is presented and proposed as a means of 68 accommodating clonal expansions. The threshold fission rate at which diffusion fails 69 to accommodate new crypts, and which may promote polyp growth, is defined. 


\section{Introduction}

71 The development of epithelial cancers is defined by mutations that become fixed and

72 expand within host tissues. In human colon somatic variants become fixed only if

73 they outcompete wildtype neighbours to successfully populate entire colonic crypts

74 (1). The subsequent process of expansion is mediated by a crypt replication process,

75 termed fission (2-4). Where clones can be visualised in situ, fission rates can be

76 directly calculated from changes in clone size distributions with age (5-11). These

77 studies confirm a low homeostatic fission rate in adult colon. In contrast, the

78 development of focal neoplastic disease is thought to be a consequence of elevated

79 fission rates. Most notably loss of the tumour suppressor gene APC is thought to

80 generate adenomas in this way (12-15). However, recently we demonstrated that

81 crypts with increased fission rates, due to loss of the X-linked gene STAG2, could

82 generate large expansions that appear phenotypically normal (1).

84 More widely it is recognised that many of the renewing epithelia, including colon,

85 acquire a substantial burden of cancer driver mutations while also remaining 86 apparently normal (16-18). Clonal expansions without an overt phenotype but 87 predisposing to oncogenic transformation may explain the recent interpretation that 88 colorectal cancers arise as a single expansion event when a combination of factors 89 reaches a critical threshold (19). This suggests that understanding the development 90 of cancer requires an appreciation not only of how cell and tissue level processes 91 allow mutational burden to be achieved, but also how it is configured within small or 92 large expansions.

94 There does not appear to be an increase in the net density of crypts, or of colonic 95 epithelial area, with age (20). This raises the question: how are local clonal 96 expansions arising from elevated fission rates accommodated? One explanation 97 might lie in crypt fusion. This process has recently been described in the mouse 98 small intestine and human colon and could counteract the consequences of fission $99(21,22)$. However, it remains unclear if fusion is a stochastic process occurring 100 independently of fission or if they are locally co-regulated. The latter possibility may 101 be particularly relevant to pro-oncogenic mutations as fusions at the edge of mutant 
102 patches could allow an effective local invasion of wildtype crypts with a high

103 probability that wildtype cells will subsequently be displaced.

104

$105 \mathrm{KDM6A}$ (UTX) is an X-linked gene encoding a histone demethylase that specifically 106 targets di- and tri-methyl groups on lysine 27 of histone H3 (H3K27me2/3). 107 Inactivating mutations and deletions of KDM6A have been identified in a variety of 108 human cancers including multiple myeloma, colon, bladder, prostate, 109 medulloblastoma, oesophageal and renal cancer (23-26). KDM6A is one of the 127 110 significantly mutated genes in The Cancer Genome Atlas (TCGA) study that 111 analysed 3281 tumours derived from 12 cancer types (27).

113 Here, in seeking additional cancer driver events that can be spatially visualised as 114 somatic clones, we identify mutations of KDM6A as possessing advantage in both 115 intra-crypt fixation and subsequent expansion. The large multicrypt clones resulting 116 from elevated rates of crypt fission are investigated to study the impact of expansion

117 on crypt packing and the role of crypt fusion in relieving overcrowding. The increased 118 fission rate within $\mathrm{KDM}^{-}$clones is not accompanied by an increase in crypt fusion, 119 suggesting the two processes are driven by independent mechanisms and that 120 fusion does not act to relieve local overcrowding. Instead, it is proposed that new 121 crypts generated by fission can be accommodated by localised crypt diffusion up to a 122 threshold beyond which hyperplastic and neoplastic lesions may form.

\section{Results}

\section{KDM6A-negative clones are advantaged in stem cell competition}

126 We have previously shown that visualisation of loss of $\mathrm{X}$-linked genes can be used 127 as clonal marks to quantify human colonic stem cell dynamics (1). In attempting to 128 expand this methodology to X-linked genes with cancer association, clonal loss of 129 KDM6A was identified by immunohistochemistry with two independent antibodies on 130 normal human colonic epithelia (Figure 1 A \& B).

132 Intra-crypt dynamics that describe the accumulation of clones wholly populating 133 entire crypts (WPC) from partly populated (PPC) transition forms were determined 134 for $\mathrm{KDM}^{-}$clones as previously described (1). Colonic FFPE sections from 120 
135 patients aged 21-93 years were stained to visualise KDM6A- clones. The total

136 number of crypts per en face section was determined using a neural-network based

137 image analysis tool (DeCryptICS, https://github.com/MorrisseyLab/DeCryptICS) and

$138 \mathrm{KDM}^{-}$clones manually counted (Figure 1C). Regression analysis revealed an age-

139 related increase in the frequency of WPC with slope, $\Delta \mathrm{C}_{f i x}$, of $6.0 \times 10^{-6} \mathrm{KDM}^{-} \mathrm{A}^{-}$

140 clones per year $\left(95 \% \mathrm{Cl}: 4.7-7.8 \times 10^{-6}\right)$ (Figure 1D). As expected, the frequency of

141 PPC, $C_{p a r t}$, remained constant at all ages $\left[2.6 \times 10^{-5}\left(95 \% \mathrm{Cl}: 1.9-3.4 \times 10^{-5}\right)\right]$

142 (Figure 1E). The ratio between these two values provides a means of comparing the

143 effect of different gene-specific mutations on stem cell dynamics that is independent

144 of the mutation rate (1). The value obtained for $\Delta \mathrm{C}_{\text {fix }} / \mathrm{C}_{\text {part }}$ was $0.23(95 \% \mathrm{Cl}: 0.16-$

145 0.34) around 4.5-fold higher than that for neutral marks and indicates a competitive

146 advantage (Figure 1F). The advantage conferred by KDM6A loss was expressed as

147 a probability of replacement $\left(P_{R}\right)$ at each round of stem cell replacement whereby

148 values above 0.5 (neutral probability of loss or gain of a mutant stem cell) identify

149 positive bias. The $\mathrm{P}_{\mathrm{R}}$ for KDM6A was 0.76 (95\% Cl: 0.60-1.0).

\section{KDM6A-negative clones expand by 5-fold increased crypt fission}

152 Expansion of individual $\mathrm{KDM}^{-}$clones was recognisable as large patches that were 153 frequently comprised of more than 10 crypts (Figure 2A). To confirm the clonal origin

154 of such patches, we used laser capture dissection followed by targeted sequencing 155 that covered $3.6 \mathrm{~kb}$ of exonic and flanking intronic sequence of KDM6A (24 156 amplicons), including sites frequently mutated in human cancers (Figure 2B \& Sup.

157 Figure 1). Amplicon libraries from four patches failed QC. Of the remaining seven 158 patches mutations were identified in four at mutant allele frequencies consistent with 159 patient sex and estimated stromal fraction within the captured material (Figure 2B 160 and Supplemental Table 1). Of note, the mutation found in intron 18 was present in 161 two independent patches. Furthermore, the mutations $S 1154^{*}$ and $\mathrm{W}^{* 193^{*}}$ have 162 previously been found in cancer samples (COSMIC database). These results both 163 confirm antibody specificity for KDM6A and the clonality of multicrypt patches that 164 share only a single mutation.

166 The age-related size distribution of multicrypt clones was analysed to infer the fission 167 rate associated with KDM6A loss and to compare that to those previously described 168 for neutral (MAOA and mPAS) and positively biased clonal marks (STAG2) (1). This 
169 revealed that the frequency of large clones ( $\geq 10$ crypts/patch) increases with age for

170 STAG2 and KDM6A but not for MAOA and mPAS (Figure 2C). Loss of KDM6A

171 generates a higher proportion of large patches than the other clonal marks while

172 STAG2 loss results in more clones due to a higher event rate (Figure 2D \& E). From

173 these patch size distributions, the crypt fission rate associated with loss of KDM6A

174 was calculated to be to $3.6 \%$ per year (95\% Cl: $3.2-4.1)$, approximately 5-fold higher

175 than the background homeostatic rate previously derived from neutral clonal marks

176 (Figure 2F). Consequently, in individuals over 80 years of age $13.5 \%$ of $\mathrm{KDM}^{-}$

177 clones are found as patches comprising more than 5 crypts compared to $4.8 \%$ for

178 STAG2, $1.7 \%$ for mPAS and $0.8 \%$ for MAOA.

179

\section{KDM6A- and STAG2- patches lack significant local overcrowding}

181 The probability of fission remains low even when elevated as for STAG2 and 182 KDM6A. Many single crypt clones never expand. Clones forming from those that do 183 are more likely to undergo subsequent fission as the probability scales with the 184 number of crypts in each clone. Consequently, the time taken to increase the 185 number of crypts in a specific area decreases rapidly with increasing patch size 186 (Figure 2G). For example, mathematical modelling based on the inferred fission rate 187 for KDM6A crypts indicates the median time taken to grow from 1 to 2 crypts is 19 188 years, but only 2 years to grow from 10 to 11 . Therefore, recently formed larger 189 patches might be expected to demonstrate overcrowding.

191 To test if larger patches are more densely packed, the area occupied by crypts and 192 their surrounding stroma was determined for 24 STAG2 and $20 \mathrm{KDM}^{-}$clones 193 containing ten crypts (Figure $2 \mathrm{H}$ ). The fraction of each patch occupied by stroma 194 was then calculated. Adjacent to each mutant clone three random groups of 10 195 crypts were defined as control 'patches' and similarly analysed. Comparing the 196 fraction of each patch occupied by stroma to adjacent wildtype groupings indicated a 197 slight trend towards increased packing density for STAG2 as well as KDM6A crypts 198 that failed to reach significance (Figure 2l). Considering that a lack of overcrowding 199 may stem from a decrease in crypt size, the areas of crypts were measured. This 200 revealed that $\mathrm{KDM}^{-}$crypt sections are around $\mathrm{x} 1.3$ the size of adjacent wildtype 201 crypts ( $p$-value <0.001). No difference was found between STAG2 ${ }^{-}$crypts and their 
202 wildtype neighbours (Supplemental Figure 2). Lack of overcrowding cannot be

203 attributed to reduced crypt size for either STAG2 or KDM6A loss.

205 Together these observations suggest that crypts even within relatively recent clonal

206 expansions avoid overcrowding to largely achieve ambient density.

\section{Evidence for crypt fusion}

209 The lack of overcrowding in $\mathrm{KDM}^{-}$clones suggests a mechanism counteracting 210 the localised increase in fission. An opposing process of crypt fusion has been 211 recognised in the mouse (21). A homeostatic human fusion rate has recently been 212 estimated and proposed as a mechanism to relieve local strain (22). This estimate 213 was based on a calculation of fission and deriving a fusion rate assuming 214 equivalence in the rate of both processes. Clearly on a tissue wide basis such a 215 balance of rates could act to maintain constant crypt density. However, local 216 increases in fission rates in advantaged clonal expansions can only balance if fission 217 and fusion are coordinately regulated by local cross talk.

219 We first sought confirmation that fusion occurs. The evidence in human epithelium is 220 based on the presence of branched crypts within which one branch is marked by clonal loss of mitochondrial CCO activity while the other is not. These are interpreted 222 as transition intermediates in an active crypt fusion process (22). Analysis of en face 223 tissue sections stained to visualise clonal mPAS positivity as well as loss of STAG2 224 and KDM6A confirmed the existence of rare heterotypic branched forms in normal 225 human colonic epithelium. Analysis of over $2 \times 10^{6}$ crypts in sections from 80 226 individuals containing $\mathrm{mPAS}^{+}$clones identified 32 candidate $\mathrm{mPAS}^{+}$branched forms 227 that were either mixed (mutant and wild type, M/W) or fully mutant (M/M) (Figure 3A).

228 Of the 13 with mixed staining (M/W) the positive epithelium was always segregated 229 into one branch and not the other.

231 An alternative interpretation of branched crypts is that they are intermediate fission 232 forms and that heterotypic staining arises due to mutations occurring or segregating 233 into a single branch (Figure 3B). We formally considered this possibility using the 234 fusion duration estimate derived by Baker and colleagues as well as our previous 235 estimates of de novo mutation probability and clone fixation rates that together 
236 determine the frequency of monoclonal crypts present in individuals of different age

$237(22,28,29)$. Within the relatively small number of branched crypts present none are 238 predicted to contain monoclonal crypt branches by either mechanism (Figure 3C).

239 This suggests that heterotypic branched crypts represent genuine intermediates

240 participating in an active fusion process. Noting that the bulk of branched crypts are

241 unstained and can represent intermediates in either fusion or fission we propose the

242 agnostic term FUFI (Fusion or Fission) to describe these transition forms.

\section{Crypt fusion and fission are regulated independently}

245 To calculate crypt fusion rates heterotypic and homotypic FUFI forms were first

246 evaluated for STAG2 and KDM6A loss by scoring around $3.9 \times 10^{6}$ and $1.8 \times 10^{6}$

247 crypts from 53 and 102 individuals each, respectively. In total, over 28,000 FUFIs

248 were evaluated. This identified 151 FUFIs with STAG2 loss of 18,928 clones

249 analysed and 63 FUFIs with KDM6A loss of 5,353 clones analysed (Figure 3D).

250 These could be found as single events or as part of multicrypt clones (Figure 3E).

251

252 Importantly, while all M/W FUFls are considered fusions and are directly observable,

$253 \mathrm{M} / \mathrm{M}$ FUFIs can be either fissions or fusions. The fusion rate is potentially accessible

254 if the relative contribution of M/M FUFI events to the total number of fusions (termed

$255 k$ ) can be determined. The value for $k$ is calculable on the basis that fusions are

256 classed as either $\mathrm{M} / \mathrm{M}$ or $\mathrm{M} / \mathrm{W}$ depending on the number of $\mathrm{M}$ and $\mathrm{W}$ neighbours

257 present at the onset of fusion. To obtain this data, M/M and M/W FUFI clones were

258 identified within multicrypt clones where the status of all their neighbours could be

259 scored as $\mathrm{W}$ or $\mathrm{M}$ (totalling 4, 121 and 49 for mPAS, STAG2 and KDM6A,

260 respectively) (Figure 3 F\&G). Single branched crypts, containing clones in either

261 branch, that possessed only $\mathrm{W}$ neighbours were also scored. This analysis revealed

$262 k$ to be 0.03 for mPAS. Within the smaller clones that are characteristic of neutral

263 clonal marks such as mPAS, most fusion events are W/M leading to low values of $k$.

264 Biased clonal marks generating large expansions will have a greater contribution

265 from M/M fusions. Correspondingly the $k$ values for STAG2 and KDM6A are 0.26

266 and 0.35 , respectively (Figure $3 \mathrm{H})$. Rates of fusion using these values of $k$ can be

267 estimated by: (i) assuming equal duration (i.e. the time window during which FUFIs

268 are detectable as intermediate forms) for both fusion and fission; (ii) using the fission 
269 rate that is independently derived from changes in patch size distributions with age

270 (see methods).

271

272 This analysis indicated similar crypt fusion rates for mPAS, STAG2 and KDM6A of $2730.3 \%$ per year (95\% Cl: 0.1-0.6), 0.4\% (95\% Cl: 0.3-0.7) and 0.7\% (95\% Cl 0.3-1.4), 274 respectively that were not significantly different (Figure 3I). Comparison of mPAS 275 fission and fusion rates, (the former is $0.7 \%$ per year; 95\% Cl: 0.5-0.9) show that 276 these closely correspond. This suggests that for neutral mutations the rates of the 277 two processes are balanced and will act together to maintain constant crypt numbers 278 across the tissue as has been suggested previously (22). However, for biased 279 mutations that, by definition, show elevated fission rates there appears to be no 280 evidence for a compensatory elevation of the fusion rate. These analyses suggest 281 that fission and fusion are independent processes and not coordinately regulated.

\section{Crypt diffusion accommodates new crypts throughout life}

284 A striking feature of larger patches is that mutant crypts have over decades 285 populated the territory initially occupied by multiple crypts without a significant 286 increase in crypt density. In the absence of appreciable merging of crypts by fusion 287 this suggests local adjustments or remodelling to allow crypts to be displaced from 288 the growing focus and return to near ambient densities. With this rationale we 289 considered the possibility of random crypt movement in the form of a diffusion 290 process.

292 Translated into the colonic epithelium the density of crypts in the 'space' of the 293 surrounding stroma is the material being diffused. Each mutant clone comprises a 294 source and new crypts created by successive fission events create a perturbation in 295 local crypt density. Crypt fission thus produces a local decrease in crypt spacing 296 which is relieved by outward diffusion, notably of both the mutant and surrounding 297 wild type crypts, until a new balance is reached (Figure 4A). The system dependent 298 diffusion coefficient (change in area per unit time) can be estimated based on 299 measured crypt spacing and theoretical bounds on patch age. 
301 To find evidence supporting a diffusion type process analysis was focused on clones

302 comprising ten crypts, the largest size for which sufficient data could be obtained for

303 STAG2 and KDM6A (24 and 20 patches, respectively). For each mutant clone, and

304 for three arbitrary control groups of ten adjacent wildtype crypts, each crypt was

305 spatially mapped in $\mathrm{X} / \mathrm{Y}$ coordinates. Areas of individual crypts as well as total patch

306 area were measured and the distance between mutant clones and wild type patch

307 centroids was determined. Finally crypt domains were defined as the area occupied

308 by both crypt and surrounding stroma (Figure 4B \& C).

310 The age of each clone was inferred in tandem with the diffusion coefficient and the 311 ambient stromal fraction for each slide to define an overall tissue process that best 312 fits the data (Supplemental Figures 4 \& 5). The tissue-intrinsic diffusion coefficient 313 describes how the tissue responds to the mutant clone by dispersing the burden of 314 decreased stromal fraction into the surrounding tissue over time. For older clones we 315 expect the system to be close to the ambient density while for younger clones 316 the perturbation to the local stromal fraction may still be evident. Examples of both 317 presumptive young and old clones were readily detectable in our samples (Figure 4D 318 and Supplemental Figures 6 \& 7).

320 For a subset of 7 patches (4 for KDM6A, 3 for STAG2) a more detailed rolling 321 window analysis was performed in which the above approach was applied where 322 fields of ten crypts were moved outwards from the mutant clone. Again evidence of a 323 reduction in stromal fraction consistent with perturbation in younger clones was 324 observed (Figure 4E and Supplemental Figures 6 \& 7). The diffusion coefficient was 325 found to be 0.055 crypt domain areas/year $(95 \% \mathrm{Cl} 0.053$ - 0.057) (Figure 4F).

326 Model comparison were performed using leave-one-out cross-validation: exhaustive 327 resampling of test-train perturbations was approximated using the full Markov chain 328 Monte Carlo output to estimate pointwise out-of-sample prediction accuracy. 329 Reassuringly this yielded a significantly worse fit than the experimental comparisons 330 (Supplemental Figure 8).

332 This inferred diffusion process can be used to define the number of crypt domains 333 affected to accommodate a new clonal expansion. The denser the new equilibrium of 334 packing, the fewer crypts are perturbed to accommodate a clone of given size 
335 (Figure 4G \& H). For example, the model suggests that patches of ten $\mathrm{KDM}^{-}$

336 crypts would require 264 crypt domains to undergo a $1 \%$ reduction in their spacing,

337 while a $5 \%$ reduction would only require 52 crypt domains (Figure $4 \mathrm{H}$ ).

\section{Defining a homeostatic threshold}

340 Limited evidence suggests that there are no significant age-related changes in colon

341 length and crypt density (20). With respect just to STAG2 and KDM6A mutations the

342 relatively few new crypts arising during life could be easily accommodated by crypt

343 movement. By the time individuals exceed 75 years of age, for every $10^{5}$ crypts,

344 fission has added only approximately 200 and 290 new STAG2 and KDM6A' crypts,

345 respectively (Figure $4 \mathrm{I}$ ). However, it seems highly probable that additional genetic

346 variants will also promote fission to different degrees. The potential for diffusion to

347 locally balance this process as fission rate increases was investigated.

Simulations were performed escalating the homeostatic fission rate of $0.7 \%$ per year. When fission rates remain below approximately 11-fold above homeostasis, diffusion can generate enough space to accommodate newly generated crypts (Figure 4J).

352 Higher fission rates result in a proportion of clones reaching a threshold of maximum 353 packing density within which crypts are directly touching. This suggests a potential

354 boundary for polyp growth, that is dependent on the physical processes of fission 355 and diffusion. Interestingly, KRAS activating mutations, which we previously found to 356 accelerate fission around 10-fold (1), mostly fall just below the threshold calculated 357 here (Figure 4J and Supplemental Figure 9). Nevertheless, our model predicts each 358 KRAS-mutant clone to harbour a cumulative probability of $0.25 \%$ of growing into a 359 visible lesion 50 years after mutation acquisition. A therapeutic intervention inhibiting 360 crypt fission for 10 years could reduce this to approximately $0.05 \%$ (Figure $4 \mathrm{~K}$ ).

\section{Discussion}

363 Several studies have shown that advantaged mutations can generate large epithelial 364 expansions (17). In the normal adult colon this is mediated by crypt fission. Perhaps 365 because this is an infrequent event there has been little consideration of the 366 compensatory mechanisms that allow newly made crypts to be accommodated 367 within a fully formed epithelium. However, here and previously it has been shown 368 that biased mutations can significantly increase crypt fission rates to generate large 
369 crypt expansions that are appropriately distributed within the tissue (1). In contrast 370 increased glandular fission rates are also known to drive the overgrowth of 371 adenomas and CRCs suggesting that differences in the rate of fission or the 372 response to it must differ between normal and neoplastic tissues (12-15).

374 In considering the epithelial responses that compensate for elevated fission rates we

375 first validated a new clonal mark, based on loss of KDM6A, that displayed advantage 376 in the process. This together with STAG2 provided two gene specific assays with 5377 and 3-fold increase in homeostatic fission rates. Comparing the configuration of the 378 size and frequency of clones for the two genes demonstrates the different strategies 379 by which age-related mutational burden can be achieved. STAG2 has the higher 380 mutation rate and generates many relatively small clones while KDM6A generates 381 fewer but larger expansions. A corollary of the exponential growth of patches as their 382 size increases is that larger patches will tend to be the most recent and therefore 383 most likely to contain evidence of local adaptation to accommodate new crypts.

385 The recently recognised process of crypt fusion offers a potential mechanism to 386 compensate for fission events (21). Assuming they had equal rates in homeostasis 387 they could effectively balance crypt numbers on a population basis (22). In 388 considering fusion as a mechanism to accommodate new crypts, a baseline estimate 389 was first established here and found to approximate that for fission. However, no 390 upregulation of fusion accompanying the local expansions resulting from STAG2 and 391 KDM6A mutation was found. Conceivably other mutations may impact fusion to ease 392 local packing but it does not appear necessary to do so.

394 Multicrypt clones that form over decades inevitably populate the territory previously 395 occupied by multiple independent crypts. In seeking to understand this dispersal we 396 looked for and found evidence of a diffusion-type process in a subset of clones. 397 These are consistent with a recent clonal expansion 'caught in the act' of being 398 restored to an ambient crypt density. The behaviour captured probably reflects a 399 passive dispersal mechanism rather than actual diffusion and must be accompanied 400 by some level of stromal turnover. 
402 The diffusion coefficient defines the rate of movement of crypt domains and the size

403 of the larger impacted zone that is required to absorb new crypts. Parameterising the

404 process allows testing of the robustness of the tissue to deal with localised 405 accelerated growth conferred by biased mutations such as STAG2 and KDM6A. 406 From this analysis the homeostatic dispersal mechanism seems over engineered 407 and able to accommodate increased fission rates of more than 10-fold above 408 baseline. Even for mutations that generate higher crypt fission rates only the fastest 409 growing clones would overgrow the available space. For example, around 5\% of 410 clones carrying a gene mutation that confers a 19-fold increase in fission rate would 411 reach a threshold where they lack a stromal domain between crypts and overgrow 412 the available space.

414 The actual threshold at which recent clonal expansions become recognisable as 415 pathologies may be lower than the extreme one applied here. However, the 416 implication remains that the distinction between phenotypically normal clones and 417 those forming overt pathologies may be determined solely by a probabilistic process 418 in which a recent succession of fission events overcome homeostatic dispersal 419 mechanisms.

421 Activating mutations of KRAS have been described in normal colonic epithelium. 422 Using targeted sequencing we previously identified high mutant allele frequencies for 423 KRAS G12 and G13 mutations in a proportion of patients and inferred that a 10-fold 424 increase in fission rate was required to achieve them. It is intriguing that activating 425 mutations of KRAS come close to achieving the fission rates capable of reaching the 426 extreme threshold defined here. If the actual threshold is lower, or if different KRAS 427 mutations confer different fission rates, then a proportion of such clones will 428 overgrow. Of note, KRAS is commonly mutated in a broad spectrum of benign and 429 premalignant pathologies such as serrated lesions and adenomas that may arise at 430 least in part due to this dispersal threshold being reached (30-34).

432 It is known that a proportion of adenomas spontaneously regress when observed in 433 longitudinal studies (35-37). One plausible explanation for such phenomena is that 434 these lesions first develop due to reaching the threshold resulting in local 435 overcrowding but are transient because of ongoing crypt dispersal. 
437 Loss of function mutations affecting the APC tumour suppressor gene are also

438 initiated and expanded by glandular fission (12-15). Mutation of both APC and 439 KRAS is frequently found in CRCs. The two pathways activated are known to 440 interact at the molecular level (38). It is likely their combined activation will also 441 synergise to further elevate gland fission rate and promote overgrowth as fully 442 neoplastic CRCs develop.

444 Clonal expansions without an overt phenotype but predisposing to oncogenic 445 transformation may explain the interpretation that colorectal cancers arise as a single 446 expansion event when a combination of factors reaches a critical threshold (19). This 447 'Big Bang' model requires that most truncal mutations required for neoplastic 448 transformation are acquired within a single cell. Accumulating such multiple hits will 449 be facilitated probabilistically by the growth of large epithelial expansions that act to 450 increase the population available for secondary mutation. However, the trigger point 451 for transformative growth may depend on additional non-mutational stochastic 452 processes of which achieving a growth rate that overruns local dispersal 453 mechanisms is one.

455 Obesity, a known risk factor for CRC, is known to be accompanied by an increase in 456 crypt fission rate (39). More specifically diets deficient for methyl donors are known 457 to reduce crypt fission rates in the mouse (40). An implication of the colon having the 458 capacity to absorb many more new crypts than are produced by homeostasis is that 459 modest and time limited reductions in the fission rates resulting from pro-oncogenic 460 mutations may not only slow the growth of lesions but prevent them from forming at 461 all.

\section{Materials and Methods}

\section{Human tissue}

465 Normal colon tissue samples were obtained from both Addenbrooke's Hospital

466 Cambridge and Norfolk and Norwich University Hospital under full local research

467 ethical committee approval (Approval document IDs 15/WA/0131 \& 17/EE/0265 as

468 well as 06/Q0108/307 \& 08/H0304/85, respectively) according to UK Home Office 
469 regulations. A total of 228 individuals were included in the study with an age range of

470 8-93 years. Colectomy specimens were fixed in 10\% neutral buffered formalin. From

471 areas of tissue without macroscopically visible disease mucosal sheets were

472 removed from the specimens and embedded en face in paraffin blocks. For standard

473 stainings, sections were cut from each sample at $5 \mu \mathrm{m}$ thickness and mounted onto

474 charged glass slides.

475

\section{6 mPAS staining}

477 Sections were dewaxed and rehydrated on a Leica Multistainer ST5020 before 478 washing in $0.1 \mathrm{M}$ Acetate buffer $\mathrm{pH} 5.5$ at $4 \stackrel{\circ}{\circ} \mathrm{C}$ for 5 minutes. This was followed by 479 oxidation in $1 \mathrm{mM}$ sodium periodate buffer at $4^{\circ} \mathrm{C}$ for 10 minutes before washing in $4801 \%$ glycerol for 5 minutes. Then, three washes were performed in ultra-pure water 481 for 5 minutes in total, followed by staining in Schiff's reagent for 15 minutes. Sections 482 were then washed again in ultra-pure water and counter-stained in Mayer's 483 Haematoxylin for 40 seconds. After another wash in ultra-pure water and brief 484 blueing in tap water and a final rinse in ultra-pure water was performed. This was 485 followed by dehydration, clearing and mounting in DPX on the same Leica 486 Multistainer.

487

\section{Immunohistochemistry}

489 Standard protocol

490 Sections were dewaxed and rehydrated on a Leica Multistainer ST5020. This was

491 followed by heat induced epitope retrieval in citrate buffer (10 mM sodium citrate, $492 \mathrm{pH6}$ ) in a medical pressure cooker and washing in PBS. All subsequent washes 493 were 3 x 5 min in PBS-T (PBS with 0.05\% Tween-20). After a 15 min incubation with $4943 \% \mathrm{H}_{2} \mathrm{O}_{2}$ in methanol and a wash, slides were incubated with blocking buffer (PBS-T 495 with 10\% Donkey Serum, Dako) for $30 \mathrm{~min}$. This and all following incubations were 496 performed at room temperature in a humidified slide box. Slides were then incubated 497 with the primary antibody (see table 1 in appendix) for one hour at room temperature 498 or overnight at $4^{\circ} \mathrm{C}$. After a wash, slides were incubated with the secondary antibody 499 (biotin-SP-conjugated AffiniPure donkey anti-mouse, anti-rabbit or anti-goat, Jackson 500 ImmunoResearch, all 1:500 in PBS-T) for $40 \mathrm{~min}$. Following a wash, slides were 501 incubated with Vectastain ${ }^{\circledR}$ Elite ${ }^{\circledR}$ ABC reagent (Vector Laboratories) for $40 \mathrm{~min}$. 
502 This was followed by a final wash and immunoperoxidase detection using a liquid

$503 \mathrm{DAB}$ + substrate chromogen system (Dako). Finally, sections were counterstained

504 with Mayer's Haematoxylin, dehydrated and mounted in DPX on the Leica

505 Multistainer.

506

507 Adaptations for laser capture slides

508 For sections for laser capture microdissection (LCM) the above protocol was 509 modified as follows: Tissue was cut at $10 \mu \mathrm{m}$ thickness onto UV-irradiated PEN 510 membrane slides (ZEISS). Heat induced epitope retrieval was performed in citrate 511 buffer in a water bath at $76{ }^{\circ} \mathrm{C}$ for 16 hours. Counterstaining with Mayer's

512 Haematoxylin was performed manually for 15 seconds followed by blueing in tap 513 water for 1 minute and drying at room temperature. Slides were stored at room 514 temperature (short-term) or $-20 \stackrel{\circ}{\circ}$ (long-term).

\section{LCM and sequencing of KDM6A-negative patches}

517 LCM and DNA extraction

518 On stained FFPE human colonic tissue sections, crypts were harvested into lids of $5190.2 \mathrm{~mm}$ radius PCR tubes using a Leica LMD7000 Laser Microdissection System. 10 $520 \mu \mathrm{l}$ of Proteinase $\mathrm{K}$ solution from the Arcturus ${ }^{\circledR}$ PicoPure® DNA Extraction Kit 521 (ThermoFisher) were added and tubes centrifuged in a mini-centrifuge for PCR 522 tubes. Following lysis for $3 \mathrm{~h}$ at $65^{\circ} \mathrm{C}$ in a standard PCR block the enzyme was 523 inactivated by incubation at $95^{\circ} \mathrm{C}$ for $10 \mathrm{~min}$. Samples were kept at $4 \stackrel{\circ}{\circ} \mathrm{C}$ until PCR.

\section{Assessment of DNA quality}

526 For assessment of optimal amplicon size, DNA extracted from FFPE sections as 527 described above was diluted to equivalents of 5, 20 and 100 crypts and used for $528 \mathrm{PCR}$ reactions containing primers at $1 \mu \mathrm{M}$ concentration (see table 2 in appendix), $5290.5 \mathrm{mM}$ dNTPs (New England BioLabs), 5 l 5X Phusion® HF Reaction Buffer (New 530 England BioLabs), $1 \mathrm{U}$ of Phusion® High-Fidelity DNA Polymerase (New England 531 BioLabs) and nuclease-free $\mathrm{H}_{2} \mathrm{O}$ (Ambion) to make up a volume of $25 \mu \mathrm{\mu}$. PCR 532 cycling was performed at $95{ }^{\circ} \mathrm{C}$ for 2 min for one cycle, followed by 35 cycles at 95

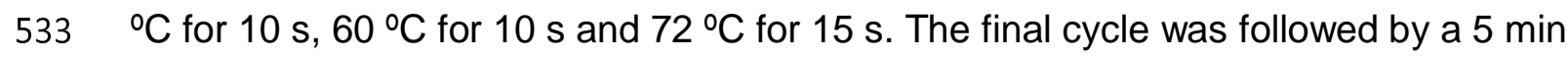
534 extension at $72^{\circ} \mathrm{C}$. 
536 Pre-amplification

537 Due to low quantities of input DNA a pre-amplification PCR was performed. All

538 primers were designed using Primer3 (41). They included Fluidigm CS1 and CS2 539 tags and were ordered from Sigma (see appendix table 3). Reactions were 540 performed in multiplex primer groups with primers each at $1 \mu \mathrm{M}$ concentration (group 541 1: primers $1,4,7 \ldots$ group 2: $2,5,8 \ldots$ group3: 3,6,9...). The LCM DNA sample was 542 diluted such that each PCR reaction contained $10 \mu$ LNA sample. In addition, each 543 reaction contained $0.5 \mathrm{mM}$ dNTPs (New England BioLabs), $5 \mu \mathrm{l}$ X Phusion® HF 544 Reaction Buffer (New England BioLabs), $1 \cup$ of Phusion® High-Fidelity DNA 545 Polymerase (New England BioLabs) and nuclease-free $\mathrm{H}_{2} \mathrm{O}$ (Ambion) to make up a 546 volume of $25 \mu \mathrm{l}$. PCR cycling was performed at $95^{\circ} \mathrm{C}$ for 2 min for one cycle, 547 followed by 35 cycles at $95^{\circ} \mathrm{C}$ for $10 \mathrm{~s}, 60^{\circ} \mathrm{C}$ for $10 \mathrm{~s}$ and $72^{\circ} \mathrm{C}$ for $15 \mathrm{~s}$. The final 548 cycle was followed by a $5 \mathrm{~min}$ extension at $72^{\circ} \mathrm{C}$. Samples were then treated with 549 ExoSAP-IT enzyme (2 $\mu$ l of enzyme for $5 \mu$ of sample, ThermoFisher) at $37^{\circ} \mathrm{C}$ for $55015 \mathrm{~min}$ followed by a $15 \mathrm{~min}$ inactivation at $80^{\circ} \mathrm{C}$. Samples were then diluted 1:10 in 551 DNA Suspension Buffer (Teknova) and stored at $4{ }^{\circ} \mathrm{C}$ until further processing.

552 To obtain adequate amounts of DNA for sequencing, the pre-amplified products 553 were further amplified using the Fluidigm Access Array ${ }^{\mathrm{TM}}$ according to the supplier's 554 protocol

556 Barcoding and Next-generation sequencing

557 A unique Fluidigm barcode was added to each sample by PCR in $10 \mu$ reactions 558 using the Fast Start High Fidelity PCR System (Roche) containing: $400 \mathrm{nM}$ 559 barcoding primers, $1 \mu$ diluted PCR product, 1 X Fast Start HF buffer without $\mathrm{MgCl}_{2}$, $5604.5 \mathrm{mM} \mathrm{MgCl}$, 5\% DMSO, $0.2 \mathrm{mM}$ dNTPs each, $0.05 \mathrm{U} / \mu \mathrm{l}$ High fidelity enzyme mix 561 and nuclease-free $\mathrm{H}_{2} \mathrm{O}$ (Ambion) to make up a reaction volume of $10 \mu \mathrm{l}$. PCR cycling 562 was performed using the protocol: 95으 for $10 \mathrm{~min}$ followed by 15 cycles at $95^{\circ} \mathrm{C}$ for $56315 \mathrm{~s}, 60^{\circ} \mathrm{C}$ for $30 \mathrm{~s}$ and $72{ }^{\circ} \mathrm{C}$ for $60 \mathrm{~s}$. The final cycle was followed by a 3 min 564 extension at $72{ }^{\circ} \mathrm{C}$. Samples were then pooled, purified using a Clean \& 565 Concentrator Kit (Zymo Research) and primer dimers eliminated by broad range 566 (200-400 bp) size selection using a PippinBlue (Sage Science). Samples from one 567 Fluidigm Access Array ${ }^{\mathrm{TM}}$ were sequenced on one lane using 150 bp paired-end 568 sequencing with $10 \%$ PhiX in-house on the Illumina MiSeq platform. 
$570 \quad$ KDM6A mutation calling

571 Fastq files were converted to .BAM files and analysed using a PERL script (available

572 upon request) by Dr Richard Kemp. Briefly, from the bulk of reads the script first 573 identifies reads corresponding to the amplicons of interest by pulling out reads

574 starting and finishing with the expected sequence as well as containing an expected

575 stretch of sequence in the middle. For these reads, at every nucleotide position

576 outside of the primer sequence, the number of reads corresponding to the reference

577 genome as well as the number of reads containing a base change at that position

578 are recorded. This enables calculation of the noise at every position. Candidate

579 mutations were identified when the mutant allele frequency was either $>4 x$ the mean

580 of the noise at that position or $>3.29 \times$ the standard deviation at that position $(\mathrm{p} \leqq$

581 0.001). True mutations were called if they were present in all samples originating

582 from the same patch in serial sections but absent in all wild-type samples from the

583 same sections. This analysis was complemented by manual inspection of reads

584 using the Integrative Genomics Viewer by the Broad Institute (42).

586 KDM6A clone data acquisition

587 Individual clones and patches

588 WPC and PPC numbers as well as multicrypt patch sizes were manually scored in 589 stained tissue sections using a standard brightfield microscope. Sections were 590 scanned at 20X using a Leica Aperio AT2 scanner and stored as .svs files on a 591 CRUK Cambridge Institute server. Scans were examined online using the Aperio 592 eSlide Manager (Leica) or downloaded and annotated using QuPath (43). To count 593 the total number of crypts on each section, .svs files were analysed with the 594 DeCryptICS neural network developed by Dr Edward Morrissey and Dr Doran 595 Khamis (https://github.com/MorrisseyLab/DeCryptICS, manuscript in preparation).

597 Quality control

598 Within the dataset two individuals aged 37 years with extreme average patch sizes 599 were identified as outliers with respect to that measure and not included in 600 subsequent analyses of patch sizes, fusion rates and newly generated crypts.

601

602 Scoring crypt fusion 
603 To score crypt fusion, the term 'FUFI' was invented to agnostically refer to structures

604 that could represent a crypt undergoing fusion or fission in en face human colonic

605 tissue sections. A FUFI was defined as two crypts joined together without any visible

606 gap but with two clearly distinguishable lumina. For stained sections, FUFIs can

607 consist of two wild type crypts (WT FUFI), two mutant crypts (mut FUFI) or a mutant

608 and wild type crypt (M/W FUFI). To count the total number of FUFIs on each section,

609 .svs files were analysed with DeCryptICS followed by manual annotation in QuPath

610 to classify FUFIs as WT, M/M or M/W.

612 Measurement of crypt packing and stromal fraction

613 Crypt domains were defined as a crypt and its surrounding stroma. Crypt domains

614 were drawn onto scans of tissue sections (.svs files) using QuPath software,

615 whereby lines were placed onto the stroma centrally between neighbouring crypts.

616 Initially, to measure the stromal fraction in STAG2- and KDM6A-mutant patches

617 comprising 10 crypts (24 and 20 patches, respectively), the total area of crypts was

618 divided by the total area of crypt domains within the patch. The same measurements

619 were also performed for adjacent groups of 10 wild type crypts, totalling three per

620 mutant patch.

621 To obtain measurements for inference of the diffusion coefficient, crypt areas, $\mathrm{x}$ - and

$622 y$-coordinates, total area of crypt domains as well as the distance to the centroid of

623 the mutant patch, $r$, were obtained for the mutant as well as surrounding wild type

624 patches. In addition, for 7 patches (3 STAG2, 4 KDM6A) the same analysis was also

625 performed as a rolling window, moving outwards from the mutant patch. This

626 included, for each mutant patch, surrounding two areas each comprising 3 mutant

627 and 7 WT, 2 mutant and 8 WT and 1 mutant and 9 WT crypts as well as five

628 surrounding WT patches at varying distances (combined: 1 data point for the mutant

629 patch and 11 for surrounding patches).

632 Mathematical inference of stem cell dynamics and crypt fission

633 The stem cell dynamics and fission rate associated with loss of KDM6A were

634 mathematically modelled as previously described (1). This includes modelling of

635 mutation acquisition as well as competition with other stem cells in the crypt, which 
636 can result in clone fixation. Subsequent crypt fission was modelled as a Yule-Furry

637 pure birth process.

638

639 Mathematical modelling of crypt fusion

640 See mathematical appendix.

642 Mathametical modelling of crypt diffusion

643 See mathematical appendix.

645 Acknowledgements

646 The authors thank the Biobanking, Histology and Genomics cores at the CRUK 647 Cambridge Institute for technical support. The authors acknowledge the contribution 648 and support to this project provided by the Norwich Research Park BioRepository 649 (Human Tissue Authority licence number 11208; NRES REC nos 06/Q0108/307 \& 650 08/H0304/85+5), a facility supported by the BBSRC, the Norfolk and Norwich 651 University Hospitals NHS Foundation Trust and the University of East Anglia. 652 Equally, the authors acknowledge contribution and support provided by the 653 Addenbrooke's Human Research Tissue Bank (NRES REC nos 15/WA/0131 \& 654 17/EE/0265) that is supported by the NIHR Cambridge Biomedical Research Centre. 655 Funding for this project was provided by a Wellcome Trust Grant (103805), Cancer 656 Research UK, a Wellcome Trust 4-year Ph.D. studentship and a Medical Research 657 Council Computational Biology Fellowship (MC_UU_12025).

\section{References}

660 1. A. M. Nicholson, et al., Fixation and Spread of Somatic Mutations in Adult 661 Human Colonic Epithelium. Cell Stem Cell 22, 909-918.e8 (2018).

662 2. K.-M. Kim, D. Shibata, Methylation reveals a niche: stem cell succession in 663 human colon crypts. Oncogene 21, 5441-5449 (2002).

664 3. K.-M. Kim, D. Shibata, Tracing ancestry with methylation patterns: most crypts 665 appear distantly related in normal adult human colon. BMC Gastroenterol. 4, 8 666 (2004).

667 4. L. C. Greaves, et al., Mitochondrial DNA mutations are established in human 668 colonic stem cells, and mutated clones expand by crypt fission. Proc. Natl. 669 Acad. Sci. U. S. A. 103, 714-719 (2006). 
670 5. R. M. Clarke, The effect of growth and of fasting on the number of villi and 671 crypts in the small intestine of the albino rat. J. Anat. 112, 27-33 (1972).

672 6. A. B. Cairnie, B. H. Millen, Fission of crypts in the small intestine of the irradiated mouse. Cell Tissue Kinet. 8, 189-96 (1975).

7. A. Maskens, Histogenesis of colon glands during postnatal growth. Acta Anat (Basel). 100, 17-26 (1978).

8. A. P. Maskens, R. M. Duhardin-Loits, Kinetics of tissue proliferation in colorectal mucosa during post-natal growth. Cell Tissue Kinet. 14, 467-77 (1981).

9. N. A. Wright, A. Al-Nafussi, The kinetics of villus cell populations in the mouse small intestine. II. Studies on growth control after death of proliferative cells induced by cytosine arabinoside, with special reference to negative feedback mechanisms. Cell Tissue Kinet. 15, 611-21 (1982).

10. H. Cheng, C. McCulloch, M. Bjerknes, Effects of $30 \%$ intestinal resection on whole population cell kinetics of mouse intestinal epithelium. Anat. Rec. 215, 35-41 (1986).

11. W. H. S. Clair, J. W. Osborne, Crypt fission in the small intestine of the rat. Br. J. Cancer. Suppl. 7, 39-41 (1986).

12. H. S. Wasan, et al., APC in the regulation of intestinal crypt fission. J. Pathol. 185, 246-255 (1998).

13. W.-M. Wong, et al., Histogenesis of human colorectal adenomas and hyperplastic polyps: the role of cell proliferation and crypt fission. Gut 50, 212 LP - 217 (2002).

14. S. L. Preston, et al., Bottom-up Histogenesis of Colorectal Adenomas. Cancer Res. 63, 3819-3825 (2003).

15. G. R. van den Brink, G. J. Offerhaus, The morphogenetic code and colon cancer development. Cancer Cell 11, 109-117 (2007).

16. I. Martincorena, et al., Tumor evolution. High burden and pervasive positive selection of somatic mutations in normal human skin. Science (80-. ). 348, 880-886 (2015).

17. I. Martincorena, et al., Somatic mutant clones colonize the human esophagus with age. Science (80-. ). 362, 911-917 (2018). epithelial cells. Nature 574, 532-537 (2019). 
704 19. A. Sottoriva, et al., A Big Bang model of human colorectal tumor growth. Nat.

705

706

707

708

709

710

711

712

713

714

715

716

717

718

719

720

721

722

723

724

725

726

727

728

729

730

731

732

733

734

735

736 Genet. 47, 209-216 (2015).

20. G. Hounnou, C. Destrieux, J. Desme, P. Bertrand, Anatomical study of the length of the human intestine. Surg. Radiol. Anat. 24, 290-294 (2002).

21. L. Bruens, S. I. J. Ellenbroek, J. van Rheenen, H. J. Snippert, In Vivo Imaging Reveals Existence of Crypt Fission and Fusion in Adult Mouse Intestine. Gastroenterology 153, 674-677.e3 (2017).

22. A.-M. Baker, et al., Crypt fusion as a homeostatic mechanism in the human colon. Gut 68, 1986-1993 (2019).

23. Z. Zhou, et al., Loss of TET1 facilitates DLD1 colon cancer cell migration via H3K27me3-mediated down-regulation of E-cadherin. J. Cell. Physiol. 233, 1359-1369 (2018).

24. M. L. Nickerson, et al., Concurrent alterations in TERT, KDM6A, and the BRCA pathway in bladder cancer. Clin. cancer Res. an Off. J. Am. Assoc. Cancer Res. 20, 4935-4948 (2014).

25. M. L. Suvà, N. Riggi, B. E. Bernstein, Epigenetic reprogramming in cancer. Science 339, 1567-1570 (2013).

26. G. van Haaften, et al., Somatic mutations of the histone H3K27 demethylase gene UTX in human cancer. Nat. Genet. 41, 521-523 (2009).

27. C. Kandoth, et al., Mutational landscape and significance across 12 major cancer types. Nature 502, 333-339 (2013).

28. C. Lopez-Garcia, A. M. Klein, B. D. Simons, D. J. Winton, Intestinal Stem Cell Replacement Follows a Pattern of Neutral Drift. Science (80-. ). 330, 822-825 (2010).

29. H. J. Snippert, et al., Intestinal Crypt Homeostasis Results from Neutral Competition between Symmetrically Dividing Lgr5 Stem Cells. Cell 143, 134144 (2010).

30. P. Zauber, S. Marotta, M. Sabbath-Solitare, KRAS gene mutations are more common in colorectal villous adenomas and in situ carcinomas than in carcinomas. Int. J. Mol. Epidemiol. Genet. 4, 1-10 (2013).

31. M. Juárez, et al., KRAS and BRAF somatic mutations in colonic polyps and the risk of metachronous neoplasia. PLoS One 12, e0184937 (2017).

32. T. Hashimoto, et al., Superficially serrated adenoma: a proposal for a novel subtype of colorectal serrated lesion. Mod. Pathol. an Off. J. United States 
738 Can. Acad. Pathol. Inc 31, 1588-1598 (2018).

739 33. A. J. McCarthy, S. Serra, R. Chetty, Traditional serrated adenoma: an

740 overview of pathology and emphasis on molecular pathogenesis. BMJ open

$741 \quad$ Gastroenterol. 6, e000317-e000317 (2019).

742 34. F. D. E. De Palma, et al., The Molecular Hallmarks of the Serrated Pathway in

743 Colorectal Cancer. Cancers (Basel). 11 (2019).

$74435 . \quad H$. Knoernschild, Growth rate and malignant potential of colonic polyps: Early

745 results. Surg. Forum 14, 137-138 (1963).

746 36. G. Hoff, A. Foerster, M. H. Vatn, J. Sauar, S. Larsen, Epidemiology of Polyps

747 in the Rectum and Colon: Recovery and Evaluation of Unresected Polyps 2

748 Years after Detection. Scand. J. Gastroenterol. 21, 853-862 (1986).

749 37. F. Loeve, et al., National Polyp Study data: evidence for regression of adenomas. Int. J. cancer 111, 633-639 (2004).

38. W.-J. Jeong, E. J. Ro, K.-Y. Choi, Interaction between Wnt/ß-catenin and RAS-ERK pathways and an anti-cancer strategy via degradations of $\beta$-catenin and RAS by targeting the Wnt/ $\beta$-catenin pathway. npj Precis. Oncol. 2, 5 (2018).

39. A. Sainsbury, et al., Increased Colorectal Epithelial Cell Proliferation and Crypt Fission Associated with Obesity and Roux-en-Y Gastric Bypass. Cancer Epidemiol. Biomarkers Prev. 17, 1401 LP - 1410 (2008).

40. M. P. Hanley, K. Kadaveru, C. Perret, C. Giardina, D. W. Rosenberg, Dietary Methyl Donor Depletion Suppresses Intestinal Adenoma Development. Cancer Prev. Res. 9, 812-820 (2016).

41. S. Rozen, H. Skaletsky, Primer3 on the WWW for general users and for biologist programmers. Methods Mol. Biol. 132, 365-86 (2000).

42. J. T. Robinson, et al., Integrative genomics viewer. Nat. Biotechnol. 29, 24-26 (2011).

43. P. Bankhead, et al., QuPath: Open source software for digital pathology image analysis. Sci. Rep. 7, 16878 (2017). 
771 (A) Representative images of IHC for cancer-associated X-linked genes on human

772 colonic sections. Out of the 7 assessed genes, a staining pattern indicative of loss-

773 of-function mutation was only found for KDM6A (middle panel).

774 (B) Serial human colonic sections stained with two independent antibodies against

775 KDM6A: (i) human protein atlas, (ii) Cell Signaling Technology. KDM6A- crypts are

776 circled and enlarged. (C) Representative images of KDM6A- WPC (i) and PPC (ii).

777 (D) Regression analysis (red line) showing accumulation of WPC $\left(\Delta \mathrm{C}_{\text {fix }}: 6.04 \times 10^{-6}\right.$

778 per year) and 95\% confidence interval in grey. (E) Top panel: Frequency plot

779 showing age-related behaviours of KDM6A-negative WPC (circles, darker) and PPC

780 (squares, lighter). Bottom panel showing PPC only on expanded y-axis. (F) Plot

781 showing increased ratio of $\Delta \mathrm{C}_{\text {fix }} / \mathrm{C}_{\text {part }}$ for $\mathrm{KDM6A}(0.23,95 \% \mathrm{Cl}: 0.16-0.34)$ as

782 compared to the neutral markers mPAS and MAOA (replotted from (1)). Error bars =

$78395 \% \mathrm{Cl}$.

784

785

Figure 2. Molecular and phenotypic characterisation of KDM6A- patches

786 (A) Representative image of large KDM6A-negative multicrypt patch. (B) Schematic 787 shows KDM6A cDNA structure with areas covered by amplicons (yellow) and 788 annotated with mutations found in COSMIC. Mutations identified in KDM6A-negative 789 patches are indicated (red). (C) Plot showing mean number of large ( $\geq 10$ crypts) 790 patches per patient for age groups shown. (D) Histogram showing the frequency of 791 different patch sizes for mPAS, MAOA, KDM6A and STAG2 across all ages. Inset 792 shows patch size $\geq 2$ crypts on expanded y-axis. (E) Dot plot of mean clone 793 frequency plotted against mean average patch size for multicrypt patches in age 794 groups shown for mPAS, MAOA, STAG2 and KDM6A. (F) Plot showing inferred 795 fission rates/crypt/year for KDM6A compared to mPAS, MAOA and STAG2 (data 796 from (1) replotted). Error bars = 95\% Cl. (G) Simulation data showing the time in 797 years taken for transitions between patch sizes 1-2 and 10-11 for (i) mPAS, (ii) 798 STAG2 and (iii) KDM6A. Insets show 10-11 transition on expanded y-axis. (H) Image 799 showing patch selection for assessment of crypt packing density within KDM6A800 (red) and adjacent wildtype crypts (blue). (I) Box plots showing fraction of patch 801 stromal space for STAG2 and KDM6Anegative mutant and adjacent wildtype 802 patches comprising 10 crypts. 


\section{Figure 3. Crypt fission and fusion are independently regulated processes}

806 (A) Schematic and representative mPAS-stained images of three types of fusion or 807 fission forms. (B) Schematic representation of alternative origins of M/W forms. Top: 808 Hypothesis 1 (Hyp1): stem cell mutation in one branch of a crypt undergoing fission 809 followed by monoclonal coversion of that branch. Bottom: Hypothesis 2 (Hyp 2):

810 fission of a pre-existing partially populated crypt with segregation of mutant and wild 811 type epithelium into each branch, followed by monoclonal conversion. (C) 812 Comparison of $\mathrm{M} / \mathrm{W}$ event frequencies simulated for hypotheses described in (B) 813 and the observed frequency. Error bars $=95 \% \mathrm{Cl}$. (D) Bar graphs showing numbers 814 of FUFI types scored for (i) mPAS, (ii) KDM6A, (iii) STAG2. (E) Representative 815 images of FUFIs in patches: (i) KDM6A M/M FUFI at border of large patch; (ii) 816 STAG2 M/W FUFI at patch border. Inset shows enlarged FUFI. (F) Schematic 817 showing scoring of FUFI neighbours used for calculation of k. (G) Dot plot showing 818 neighbouring crypt status of patch border FUFIs (either M/M or M/W type) for mPAS, 819 STAG2 and KDM6A. Each dot represents one or more FuFis for which neighbours 820 were scored. $(\mathrm{H})$ Plot comparing the value of $k$, representing the fraction of $M / M$ 821 FUFls that are fusions, for mPAS, STAG2 and KDM6A. (I) Plot comparing the 822 derived fission and fusion rates associated with mPAS, STAG2 and KDM6A. mPAS and STAG2 fission rates correspond to data from (1), replotted. Error bars $=95 \% \mathrm{Cl}$.

\section{Figure 4. Evidence for a crypt diffusion process}

826 (A) Schematic representation of the proposed crypt diffusion process. Fission of a 827 clone results in higher local density which is then relieved by diffusion. (B) Schematic 828 representation of areas measured to assess packing of mutant patch and 829 surrounding crypts in rolling windows placed at different distances $(R)$ from centroid 830 of mutant patch. (C) Schematic showing crypt cross section with area of surrounding 831 stroma that defines a crypt domain. (D) Examples of fitted diffusion process to 832 achieve ambient density when comparing patches of 10 crypts when moving from 833 mutant clone to three adjacent control groupings. (i) STAG2, (ii) KDM6A. Points are 834 data derived, line is best fit, grey shading is $95 \% \mathrm{Cl}$ : presumed "young" patch and 835 bottom: presumed "old" patch for i) STAG2 and ii) KDM6A. (E) As (D) but with rolling 836 window data. (F) Density plot of values obtained for the diffusion coefficient in human 837 colonic epithelium. (G) Schematic representation of area of crypts affected if space is 
838 decreased by $5 \%$ or $1 \%$ surrounding a mutant patch of 10 . (Crypt numbers not to

839 scale). (H) Boxplot showing the simulated numbers of crypts affected if spacing is

840 decreased by $1 \%$ or $5 \%$ respectively due to addition of a patch of 10 mutant crypts.

841 (I) Dotplot showing median frequency of newly generated crypts for mPAS, MAOA,

842 STAG2 and KDM6A across four age bins. (J) Line graphs showing the stromal

843 fraction resulting from crypt diffusion and different crypt fission rates corresponding

844 to the homeostatic (wild-type) rate as well as those associated with STAG2, KDM6A

845 and KRAS and multiples of 11 and 12 of the WT rate. Dotted line = whitespace

846 fraction calculated from optimal hexagonal packing of circles. $R=$ distance from

847 centroid of patch in crypt domains. (K) Line graph showing results from simulations

848 to calculate the cumulative probability of a clone growing into a lesion over time.

849 Simulations were performed using the fission rate associated with KRAS activation

850 (none) and are shown alongside the results obtained for simulation inclulding

851 therapeutic crypt fission inhibition applied for a decade either immediately after

852 mutation acquisition (0-10), after 10 years (10-20) or 20 years (20-30). 
bioRxiv preprint doi: https://doi.org/10.1101/2020.07.10.193748; this version posted July 12, 2020. The copyright holder for this preprint (which was not certified by peer review) is the author/funder, who has granted bioRxiv a license to display the preprint in perpetuity. It is made available under aCC-BY-NC-ND 4.0 International license.

A

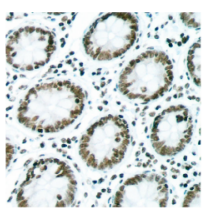

AMER1

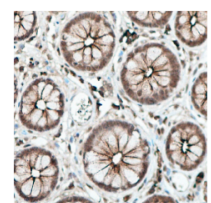

ATRX

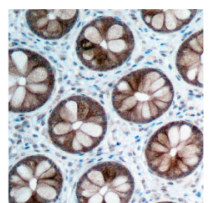

HUWE1

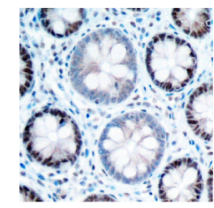

KDM6A

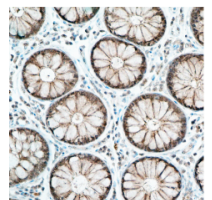

PDHA1

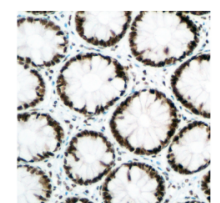

RBMX
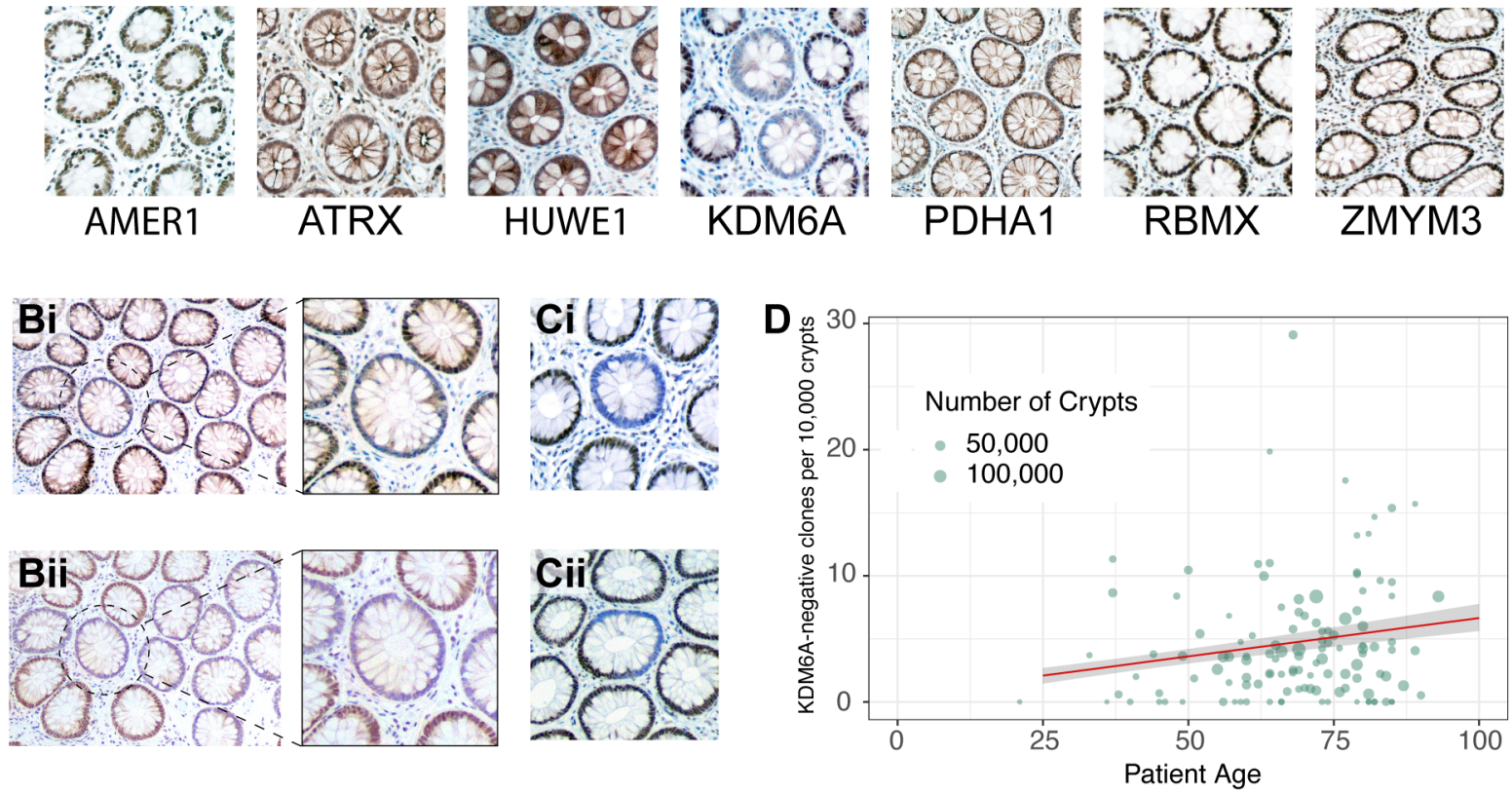

E

F
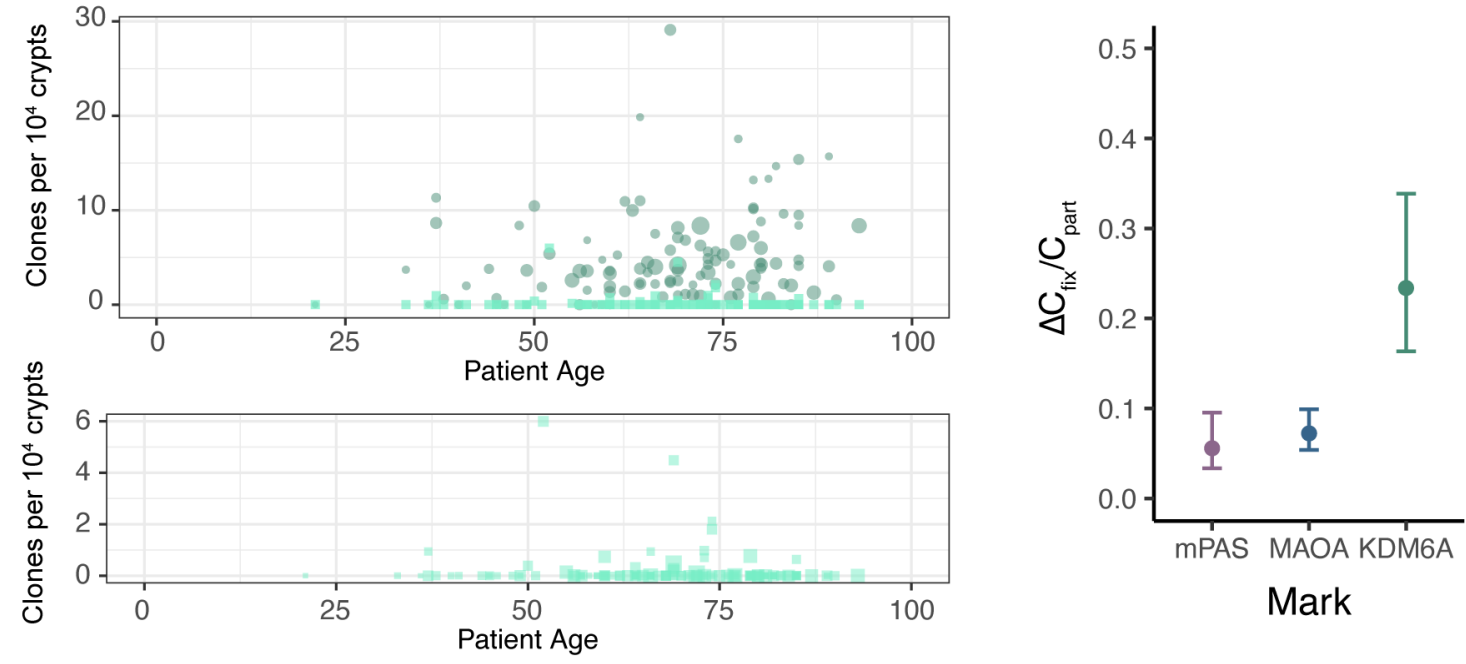
A

WT

M/W

$\mathrm{M} / \mathrm{M}$

fission or fusion likely fusion fission or fusion
C

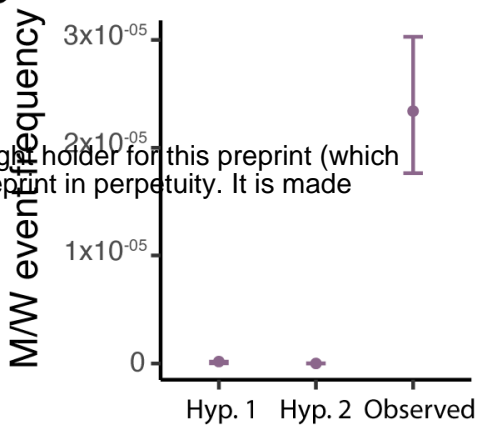

Ei

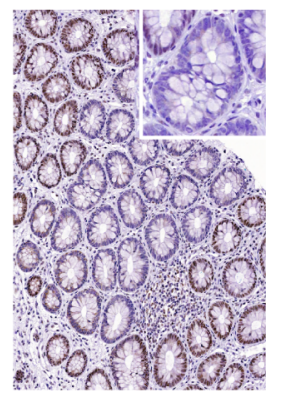

Eii

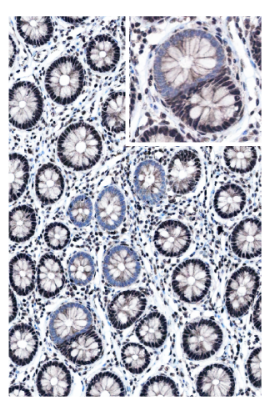

F

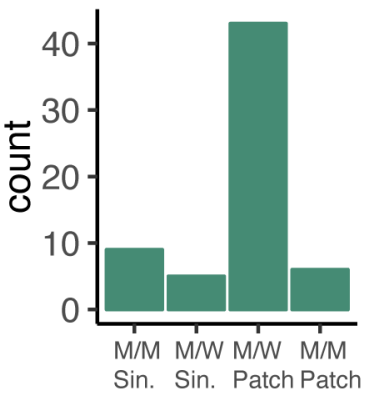

H

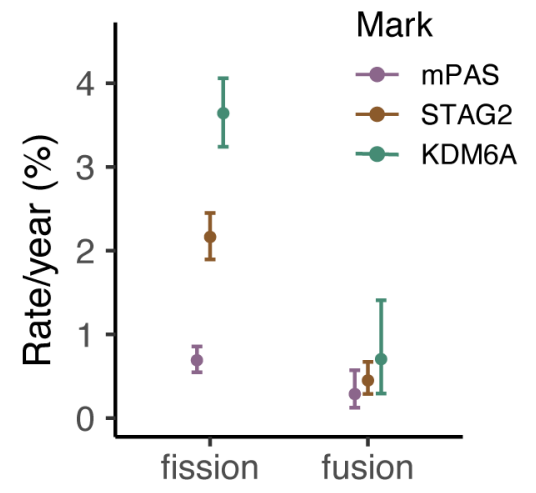

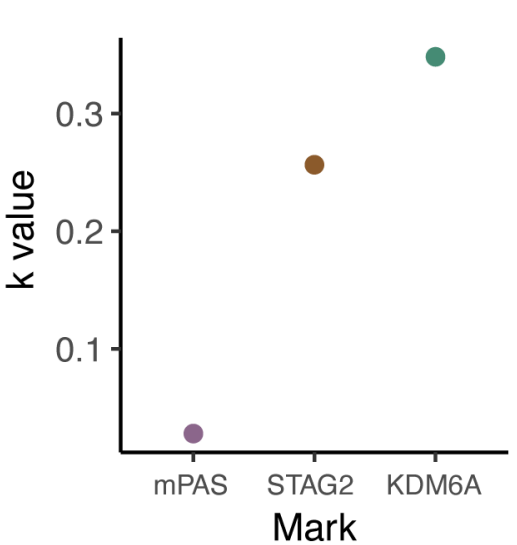

2 mut neighbours o 1 FUFI

5 WT neighbours

\section{I} fusion
G

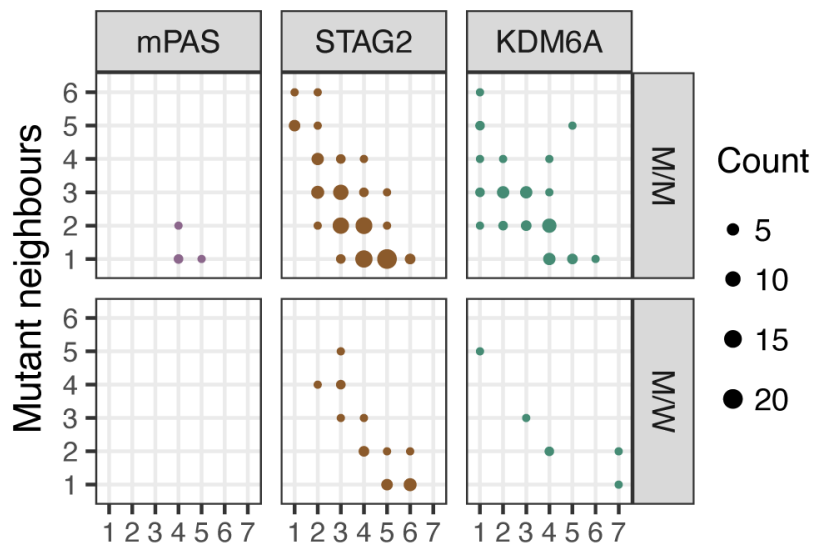

WT neighbours 
